\title{
Editorial
}

\author{
Amir H. Alavi ${ }^{1}$ - Zhihua Cui ${ }^{2} \cdot$ Amir H. Gandomi ${ }^{1} \cdot$ Xiao-Zhi Gao $^{3} \cdot$ Gai-Ge Wang $^{4} \cdot$ Meng-Hiot Lim ${ }^{5}$
}

Published online: 24 May 2018

(c) Springer-Verlag GmbH Germany, part of Springer Nature 2018

Swarm intelligence is a discipline that deals with the natural and artificial systems composed of many individuals that coordinate their activities using decentralized control and self-organization. Lately, swarm intelligence algorithms are becoming increasingly important in the face of the complexity of today's demanding applications. This issue aims to present a collection of recent advances in the swarm intelligence algorithms. On the basis of a peer-review process, six papers are accepted to be included in the thematic issue, covering various algorithms including particle swarm optimization, monarch butterfly optimization, moth search algorithm, evolutionary computation, and cuckoo search.

Surrogate assisted meta-heuristic algorithms have received increasing attention over the past years, due to the fact that numerous real-world optimization problems are computationally expensive. However, most existing surrogate assisted meta-heuristic algorithms are designed for small or medium scale problems. In the first paper of this issue, Chaoli Sun et al. propose a fitness approximation assisted competitive swarm optimizer for the optimization of large scale expensive problems. Different from regular surrogate assisted evolutionary algorithms that use a computational model for approximating the fitness, this paper uses estimates of fitness based on the positional relationship between the individuals in the competitive swarm optimizer. Empirical study on seven widely used benchmark problems with 100 and 500 decision variables show that the proposed fitness approximation assisted competitive swarm optimizer can achieve competitive performance on a limited computational budget.

$\triangle$ Meng-Hiot Lim

EMHLIM@ntu.edu.sg

Michigan State University, East Lansing, USA

2 Taiyuan University of Science and Technology, Taiyuan, China

3 University of Eastern Finland, Kuopio, Finland

4 Ocean University of China, Qingdao, China

5 Nanyang Technological University, Nanyang Avenue, Singapore
Recently, inspired by the migration behavior of monarch butterflies in nature, a metaheuristic optimization algorithm, called monarch butterfly optimization (MBO), has been proposed. In the present study by Yanhong Feng et al., a novel chaotic $\mathrm{MBO}$ algorithm (CMBO) is developed, in which chaos theory is introduced in order to enhance its global optimization ability. Here, a total of 12 one-dimensional classical chaotic maps are used to tune two main migration processes of monarch butterflies. Meanwhile, applying Gaussian mutation operator to some worst individuals can effectively prevent the premature convergence of the optimization process. The performance of the $\mathrm{CMBO}$ is verified and analyzed by three groups of large-scale 0-1 knapsack problems instances. The results obtained demonstrate that the introduction of appropriate chaotic map and Gaussian perturbation can significantly improve the solution quality together with the overall performance of the proposed CMBO algorithm. The CMBO is capable of outperforming the standard MBO and eight other state-of-the-art canonical algorithms.

The third paper by Gai-Ge Wang proposes a new kind of metaheuristic algorithm, called moth search (MS) algorithm. In nature, moths are a family of insects associated with butterflies belonging to the order Lepidoptera. In the MS method, the best moth individual is viewed as the light source. Some moths that are close to the fittest one always display inclination to fly around their own positions in the form of Lévy flights. On the contrary, due to phototaxis, the moths that are comparatively far from the fittest one will tend to fly towards the best one directly in big step. These two features correspond to the processes of exploitation and exploration of most metaheuristic optimization methods. The phototaxis and Lévy flights of the moths can be used to build up a general-purpose optimization method. In order to demonstrate the superiority of its performance, the MS method is further compared with five other up-to-date metaheuristic optimization algorithms using an array of experiments on 14 basic benchmarks. The results clearly demonstrate that the MS significantly outperforms five other methods on most of the test functions and engineering cases. 
The Generalized Vertex Cover Problem (GVCP) extends classic vertex cover problems to take both vertex and edge weights into consideration in the objective function. The GVCP results in finding a vertex subset such that the sum of vertex weights together with all the corresponding edge weights is minimized. In this paper, Shuli $\mathrm{Hu}$ et al. propose a hybrid metaheuristic algorithm to solve the GVCP (MAGVCP for short) that is based on evolutionary search and iterated neighborhood search. The algorithm uses population initializing procedure to produce high-quality solutions, applies a dedicated crossover to generate offspring solutions, and finally utilizes an iterated best chosen neighborhood search to find better solutions. Experiments carried on random instances and DIMACS instances verify the effectiveness of the proposed algorithm.

The fifth paper by Heqi Wang et al. presents a robust optimization algorithm based on the hybridization of the krill herd $(\mathrm{KH})$ and artificial bee colony $(\mathrm{ABC})$ methods and the information exchange concept. The global optimal solutions found by the proposed hybrid $\mathrm{KH}$ and $\mathrm{ABC}$ (KHABC) algorithm are considered as a neighbor food source for the onlooker bees in the ABC. Thereafter, a local search is performed by the onlooker bees in order to find a better solution around the given neighbor food source. Both methods, the $\mathrm{KH}$ and $\mathrm{ABC}$, share the globally best solutions through the information exchange process between the krill and bees. Based on the above results, the exchange process significantly improves the exploration and exploitation of the hybrid method. Besides, a focused elitism scheme is introduced to enhance the performance of the developed algorithm. The validity of the KHABC method is validated using 13 unconstrained benchmark functions, a total of 21 CEC 2017 constrained real-parameter optimization problems, and ten CEC 2011 real world problems (RWPs). The proposed method clearly demonstrates its potential for a competitive optimization tool towards solving both benchmark functions and real world problems.

Cuckoo search (CS) is a recently developed meta-heuristic, which has shown good search abilities on many optimization problems. Maoqing Zhang et al. present a hybrid multiobjective CS (HMOCS) for solving multi-objective optimization problems (MOPs). The HMOCS employs the nondominated sorting procedure and a dynamical local search. The former is helpful to generate Pareto fronts, and the latter focuses on enhancing the local search. In order to prove the capability of the HMOCS, six well-known benchmark MOPs are used in their experiments. Simulation results show that HMOCS outperforms three other multi-objective algorithms in terms of convergence, spread, and distributions.

It has indeed been a pleasure to edit this special issue. We are grateful to all the authors who submitted their papers and to the reviewers for their timely and insightful reviews that have greatly helped improve the quality of this issue. We hope that the readers find the papers collected in this thematic issue interesting and helpful. The Guest Editors acknowledge the financial supports from the Natural Science Foundation of Shanxi Province under Grant No. 201601D011045.

\section{Notes from Editor-in-Chief}

The above six articles make up the Thematic Issue on Novel Swarm Intelligence Algorithms. To further complement this thematic issue, we include two more regular issue papers on applications to complete the issue. The paper by Nodeh et. al. describes a hybrid algorithm that combines differential evolution and mutable smart bee algorithm. The work has practical significance, being applied to optimize the fabrication of piezoelectric excited millimetre-sized cantilever sensor. Such a problem usually involves constraints, and the authors show that their approach can optimize the design parameters for the sensors. Another paper by Liu et al. deals with active object recognition based on sequence of actions. This is achieved by means of camera with adjustable position and orientation. They use extreme learning machine to learn the state representations and the strategy for reinforcement learning, which is validated based on a public-domain dataset.

Publisher's Note Springer Nature remains neutral with regard to jurisdictional claims in published maps and institutional affiliations. 